\title{
Properties of Iron Primary Cosmic Rays: Results from the
} Alpha Magnetic Spectrometer

\section{Yao Chen ${ }^{a, *}$ and Mercedes Paniccia ${ }^{b}$ on behalf of the AMS Collaboration}

(a complete list of authors can be found at the end of the proceedings)

a Shandong Institute of Advanced Technology (SDIAT),

Jinan, Shandong, 250100, China

${ }^{b}$ DPNC, Université de Genève,

1211 Genève 4, Switzerland

E-mail: yao.chen@cern.ch, Mercedes.Paniccia@cern.ch

We report the observation of new properties of primary iron $(\mathrm{Fe})$ cosmic rays in the rigidity range $2.65 \mathrm{GV}$ to $3.0 \mathrm{TV}$ with 0.62 million iron nuclei collected by the Alpha Magnetic Spectrometer experiment on the International Space Station. Above $80.5 \mathrm{GV}$ the rigidity dependence of the cosmic ray $\mathrm{Fe}$ flux is identical to the rigidity dependence of the primary cosmic ray $\mathrm{He}, \mathrm{C}$, and O fluxes, with the Fe/O flux ratio being constant at $0.155 \pm 0.006$. This shows that unexpectedly $\mathrm{Fe}$ and $\mathrm{He}, \mathrm{C}$, and $\mathrm{O}$ belong to the same class of primary cosmic rays which is different from the primary cosmic rays $\mathrm{Ne}, \mathrm{Mg}$, and $\mathrm{Si}$ class.

$37^{\text {th }}$ International Cosmic Ray Conference (ICRC 2021)

July 12th - 23rd, 2021

Online - Berlin, Germany

\footnotetext{
*Presenter
} 


\section{Introduction}

Primary iron cosmic rays are the most abundant heavy nuclei beyond silicon. They are thought to be mostly produced and accelerated in astrophysical sources. Precise knowledge of the iron spectrum in the GV-TV rigidity region provides important information on the origin, acceleration, and propagation processes of cosmic rays in the Galaxy [1].

We present the precise measurement of the Fe flux in the rigidity range from $2.65 \mathrm{GV}$ to 3.0 TV based on $0.62 \times 10^{6}$ iron nuclei collected by the Alpha Magnetic Spectrometer (AMS) during its first 8.5 years (from May 19, 2011 to October 30, 2019) of operation on the International Space Station (ISS).

\section{AMS-02 Detector and analysis}

The AMS detector is a large-acceptance magnetic spectrometer, the full description of the detector is presented in [2] and references therein. The key elements used in this measurement are the permanent magnet, the nine layers of silicon tracker, L1-L9, and the four planes of time of flight (TOF) scintillation counters. Together with the permanent magnet, the silicon tracker measures the rigidity of charged cosmic rays.

In the first 8.5 years of operation AMS has collected $1.50 \times 10^{11}$ cosmic ray events. Iron events are required to be downward going and to have a reconstructed track in the seven tracker layers placed on the top and inside the magnet. The track is also required to pass through tracker L1, and for the highest rigidity region $\mathrm{R} \geq 1.2 \mathrm{TV}$, through tracker L9. Charge measurements on tracker L1, the inner tracker (L2-L8), the upper TOF, and, for R $\geq 1.2 \mathrm{TV}$, the lower TOF, and tracker L9 are required to be compatible with charge $\mathrm{Z}=26$. Details of the Iron flux analysis procedure and particularly the studies of the systematic errors can be found in [3].

The material traversed by nuclei from the top of AMS to tracker L9 is composed primarily of carbon and aluminum. The survival probabilities of Fe nuclei due to interactions in the materials were measured using cosmic ray data collected by AMS as described in [4]. The simulation of nuclear interactions has been validated with data using all AMS measured nuclear charge changing cross sections $(\mathrm{Fe} \rightarrow \mathrm{He} . . \mathrm{Mn}+\mathrm{X}$ ). Fig. 1 shows examples for $\mathrm{Fe} \rightarrow \mathrm{Cr}+\mathrm{X}, \mathrm{Fe} \rightarrow \mathrm{Si}+\mathrm{X}$, and $\mathrm{Fe} \rightarrow \mathrm{O}+\mathrm{X}$.

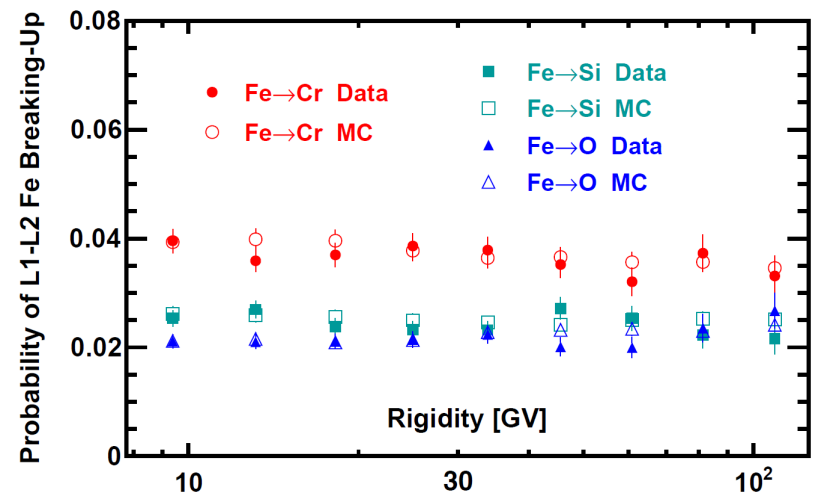

Figure 1: Comparison of the simulated and measured $\mathrm{Fe} \rightarrow \mathrm{Cr}+\mathrm{X}, \mathrm{Fe} \rightarrow \mathrm{Si}+\mathrm{X}$, and $\mathrm{Fe} \rightarrow \mathrm{O}+\mathrm{X}$ break-up probabilities between tracker L1 and tracker L2. 


\section{Properties of Iron Cosmic Ray nuclei}

Fig. 2 shows the measured Fe flux as a function of rigidity $\tilde{R}$ at the top of the AMS detector with the total errors, the sum in quadrature of statistical and systematic errors. In the figure the points are placed along the abscissa at $\tilde{R}$ calculated for a flux $\propto \mathrm{R}^{-2.7}$ [5]. For comparison, Fig. 2 also shows the latest AMS result on the oxygen flux from $[2,6]$.

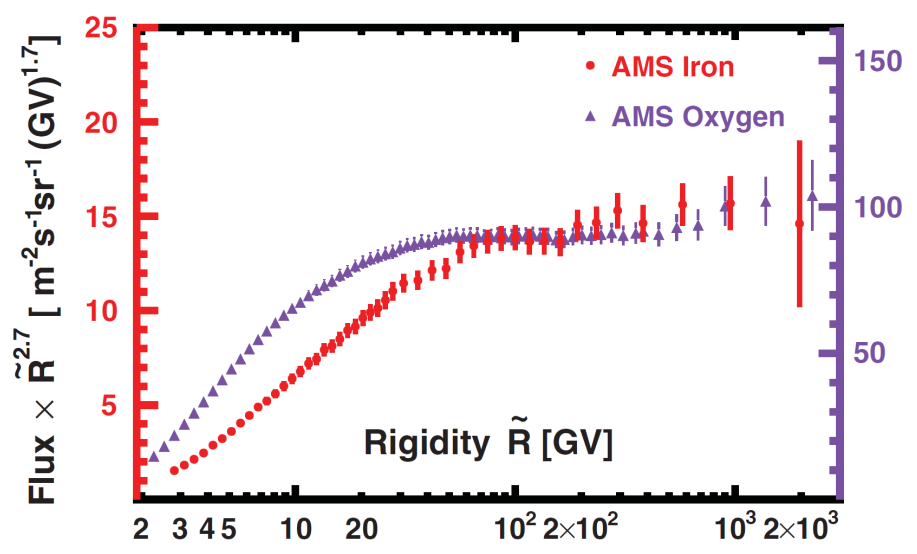

Figure 2: The AMS iron flux (red dots) and oxygen flux (violet triangles) multiplied by $\tilde{R}^{2.7}$ with total errors as a function of rigidity.

To examine the rigidity dependence of the Fe flux, the variation of the flux spectral index with rigidity was obtained in a model independent way as $\gamma=d[\log (\Phi)] / d[\log (\Phi)]$ computed over nonoverlapping rigidity intervals bounded by 7.09, 12.0, 16.6, 28.8, 45.1, 80.5, 175.0, and 3000.0 GV. The results are presented in Fig. 3 together with the spectral index of the oxygen flux from [2]. As seen, above $80.5 \mathrm{GV}$ the rigidity dependence of the iron flux and its spectral index follow the rigidity dependence of the oxygen flux and its spectral index.

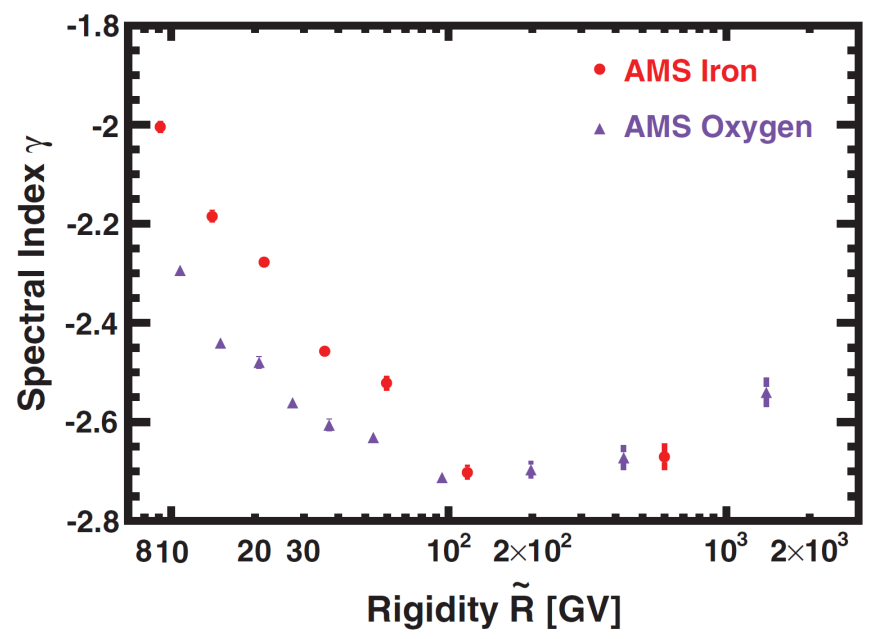

Figure 3: The AMS iron flux spectral index (red dots) and oxygen flux spectral index (violet triangles) as functions of rigidity. 
Fig. 4 shows the AMS iron flux as a function of kinetic energy per nucleon $E_{K}$ together with measurements from other experiments [7-17].

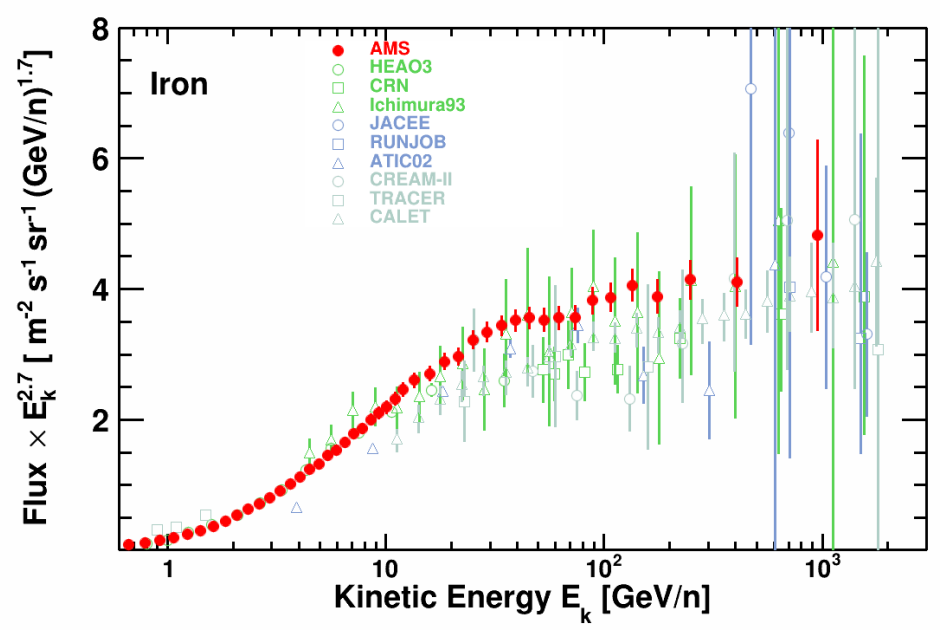

Figure 4: The AMS iron flux as a function of kinetic energy per nucleon $E_{K}$ multiplied by $E_{K}^{2.7}$ together with measurements from other experiments [7-17]. For the AMS measurement $E_{K}=\left(\sqrt{Z^{2} \tilde{R}^{2}+M^{2}}-M\right) / A$ where $Z, M$, and $A$ are the ${ }_{26}^{56} \mathrm{Fe}$ nuclear charge, mass, and atomic mass numbers, respectively. Data from CALET are taken from [17] and data from other experiments are taken from [18].

To compare the rigidity dependence of the $\mathrm{Fe}$ flux with that of $\mathrm{He}, \mathrm{C}$, and $\mathrm{O}$ primary cosmic ray fluxes, which have identical rigidity dependence above $60 \mathrm{GV}[2,6]$, the ratio of the iron flux to the oxygen flux [2], Fe/O, was computed. Fig. 5 shows the AMS Fe/O ratio as a function of rigidity with total errors together with a constant value fit above $80.5 \mathrm{GV}$. The fit yields $\mathrm{Fe} / \mathrm{O}=0.155 \pm 0.006$ with $\chi^{2} /$ d.o.f. $=8 / 11$. This, together with Fig. 2, shows that Fe belongs to the same class of primary cosmic rays as $\mathrm{He}, \mathrm{C}$, and $\mathrm{O}$.

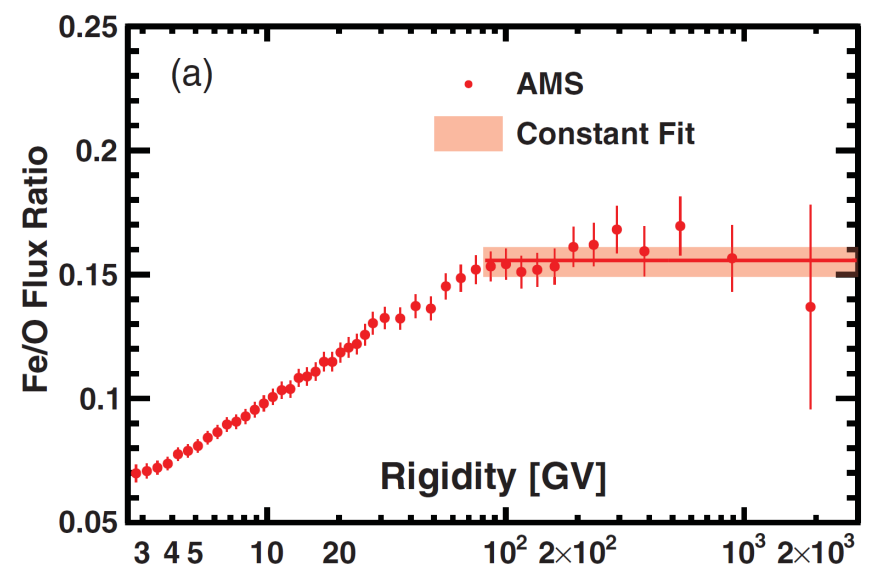

Figure 5: The AMS Fe/O ratio as a function of rigidity with total errors and with red band $(1 \sigma)$ indicating the constant value fit $\left(\mathrm{Fe} / \mathrm{O}=0.155 \pm 0.006\right.$ with $\chi^{2} /$ d.o.f. $\left.=8 / 11\right)$ above $80.5 \mathrm{GV}$.

To compare the AMS result with previous measurements, the Fe/O ratio was converted from rigidity to kinetic energy per nucleon using the procedure described in [19]. Fig. 6 shows the 
AMS Fe/O flux ratio as a function of kinetic energy per nucleon together with earlier measurements $[7,8,11,13,15,16]$. As seen, the AMS result provides an accurate measurement of the energy dependence of the Fe/O flux ratio.

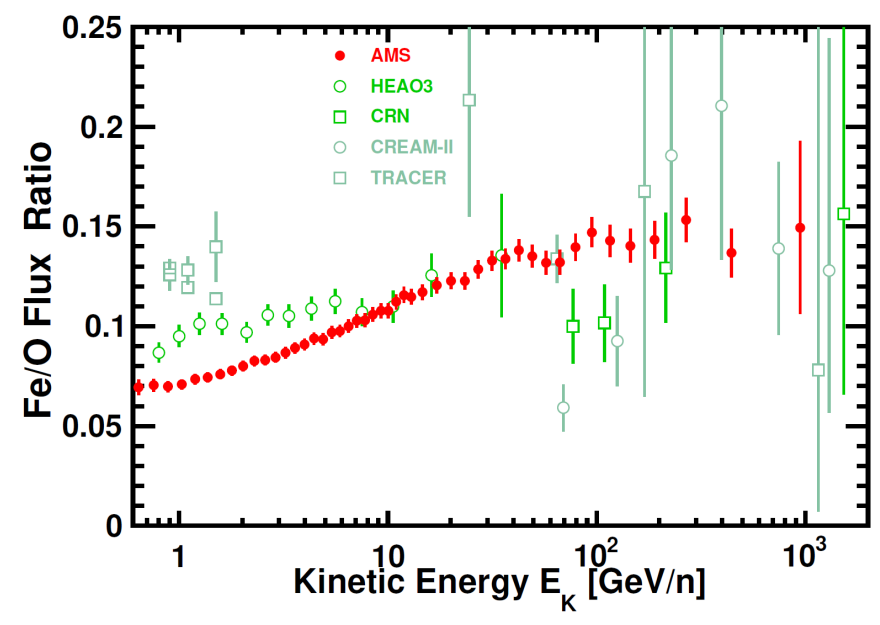

Figure 6: The AMS Fe/O flux ratio as a function of kinetic energy per nucleon $E_{K}$ together with earlier measurements. To convert the AMS measurements from rigidity to kinetic energy per nucleon we used ${ }^{56} \mathrm{Fe}$ and ${ }^{16} \mathrm{O}$.

In summary, the rigidity dependence of $\mathrm{Fe}$ above $80.5 \mathrm{GV}$ follows the rigidity dependence of O, see Fig. 2 and Fig. 5. Therefore as shown in Fig. 7, unexpectedly Fe belongs to the class of light primary cosmic rays $\mathrm{He}, \mathrm{C}$ and $\mathrm{O}[2,6]$, which is different from the rigidity dependence of the heavy primary cosmic rays $\mathrm{Ne}, \mathrm{Mg}$, and $\mathrm{Si}$ [20].

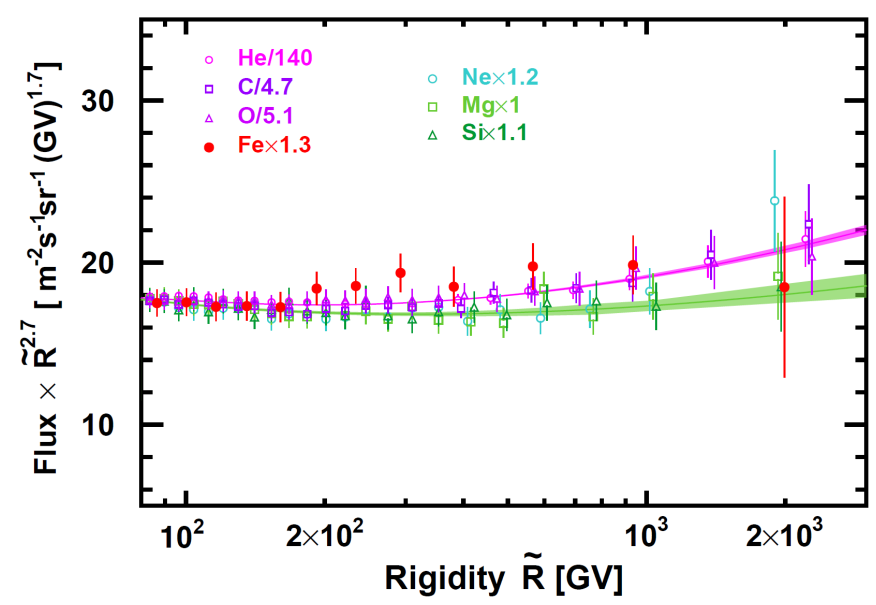

Figure 7: The rigidity dependence of the Fe spectrum compared with the rigidity dependence of the $\mathrm{He}, \mathrm{C}$, and $\mathrm{O}$ spectra and the $\mathrm{Ne}, \mathrm{Mg}$, and $\mathrm{Si}$ spectra above $80.5 \mathrm{GV}$. For clarity, the $\mathrm{He}, \mathrm{O}, \mathrm{Ne}$, and $\mathrm{Si}$ data points above $400 \mathrm{GV}$ are displaced horizontally. For display purposes only, the $\mathrm{He}, \mathrm{C}, \mathrm{O}, \mathrm{Ne}, \mathrm{Si}$, and $\mathrm{Fe}$ spectra were rescaled as indicated. The shaded areas show the fit result of Eq. (5) of [20] to He, C, and O spectra (magenta) and $\mathrm{Ne}, \mathrm{Mg}$, and Si spectra (green). 


\section{Conclusion}

We have presented the precision measurement of the cosmic ray Fe spectrum as a function of rigidity from $2.65 \mathrm{GV}$ to $3.0 \mathrm{TV}$. Above $80.5 \mathrm{GV}$ the rigidity dependence of the cosmic-ray $\mathrm{Fe}$ spectrum is identical to the rigidity dependence of the light primary cosmic-ray spectra $\mathrm{He}, \mathrm{C}$, and $\mathrm{O}$, which is different from the rigidity dependence of the heavy primary cosmic-ray $\mathrm{Ne}, \mathrm{Mg}$, and $\mathrm{Si}$ spectra. In particular, above $80.5 \mathrm{GV}$ the $\mathrm{Fe} / \mathrm{O}$ ratio is well described by a constant value of $0.155 \pm 0.006$. These are new and unexpected properties of primary iron cosmic rays.

\section{References}

[1] I. A. Grenier, J. H. Black, and A.W. Strong, Annu. Rev. Astron. Astrophys. 53, 199 (2015); P. Blasi, Astron. Astrophys. Rev. 21, 70 (2013); A.W. Strong, I. V. Moskalenko, and V. S. Ptuskin, Annu. Rev. Nucl. Part. Sci. 57, 285 (2007); A. Castellina and F. Donato, Astropart. Phys. 24, 146 (2005).

[2] M. Aguilar et al., Phys. Rep. 894, 1 (2021).

[3] M. Aguilar et al., Phys. Rev. Lett. 126, 041104 (2021).

[4] Q. Yan, V. Choutko, A. Oliva, and M. Paniccia, Nucl. Phys. A996, 121712 (2020).

[5] G. D. Lafferty and T. R. Wyatt, Nucl. Instrum. Methods Phys. Res., Sect. A 355, 541 (1995). We have used Eq.(6) with $\tilde{R} \equiv x_{l w}$.

[6] M. Aguilar et al., Phys. Rev. Lett. 119, 251101 (2017).

[7] J. J. Engelmann et al., Astron. Astrophys. 233, 96 (1990).

[8] S. P. Swordy, D. Müller, P. Meyer, J. L’Heureux, and J. M. Grunsfeld, Astrophys. J. 374, 356 (1991).

[9] M. Ichimura, M. Kogawa, S. Kuramata, H. Mito, and T. Murabayashi et al., Phys. Rev. D 48, 1949 (1993).

[10] Y. Takahashi, Nucl. Phys. B, Proc. Suppl. 60, 83 (1998); T. A. Parnell et al., Adv. Space Res. 9, 45 (1989).

[11] F. Gahbauer, G. Hermann, J. R. Hörandel, D. Müller, and A. A. Radu, Astrophys. J. 607, 333 (2004).

[12] V. A. Derbina et al., Astrophys. J. 628, L41 (2005).

[13] M. Ave, P. J. Boyle, F. Gahbauer, C. Höppner, J. R. Hörandel, M. Ichimura, D. Müller, and A. Romero-Wolf, Astrophys. J. 678, 262 (2008).

[14] A. D. Panov et al., Bull. Russ. Acad. Sci. 73, 564 (2009).

[15] H. S. Ahn et al., Astrophys. J. 715, 1400 (2010); 707, 593 (2009). 
[16] A. Obermeier, M. Ave, P. J. Boyle, C. Höppner, J. R. Hörandel, and D. Müller, Astrophys. J. 742, 14 (2011).

[17] O. Adriani et al. (CALET Collaboration), Phys. Rev. Lett. 126, 241101

[18] D. Maurin, F. Melot, and R. Taillet, Astron. Astrophys. 569, A32 (2014).

[19] M. Aguilar et al., Phys. Rev. Lett. 117, 231102 (2016).

[20] M. Aguilar et al., Phys. Rev. Lett. 124, 211102 (2020). 


\section{Full Authors List: AMS Collaboration}

M. Aguilar ${ }^{30}$ L. Ali Cavasonza ${ }^{1}$ M. S. Allen ${ }^{10}$ B. Alpat ${ }^{37}$ G. Ambrosi ${ }^{37}$ L. Arruda ${ }^{28}$ N. Attig ${ }^{24}$ F. Barao ${ }^{28}$ L. Barrin ${ }^{15}$ A. Bartoloni ${ }^{43}$ S. Başeğmez-du Pree ${ }^{18, *}$ R. Battiston ${ }^{40,41}$ M. Behlmann ${ }^{10}$ B. Beischer ${ }^{1}$ J. Berdugo ${ }^{30}$ B. Bertucci ${ }^{37,38}$ V. Bindi ${ }^{20}$ W. de Boer ${ }^{25}$ K. Bollweg $^{21}$ B. Borgia ${ }^{43,44}$ M. J. Boschini ${ }^{32}$ M. Bourquin ${ }^{16}$ E. F. Bueno ${ }^{18}$ J. Burger ${ }^{10}$ W. J. Burger ${ }^{40}$ S. Burmeister ${ }^{26}$ X. D. Cai ${ }^{10}$ M.

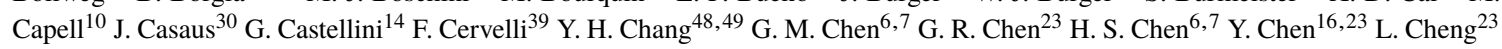
H. Y. Chou ${ }^{49}$ S. Chouridou ${ }^{1}$ V. Choutko ${ }^{10}$ C. H. Chung ${ }^{1}$ C. Clark ${ }^{10,21}$ G. Coignet $^{3}$ C. Consolandi ${ }^{20}$ A. Contin ${ }^{8,9}$ C. Corti $^{20}$ Z. Cui $^{22,23}$ K. Dadzie ${ }^{10}$ C. Delgado ${ }^{30}$ S. Della Torre ${ }^{32}$ M. B. Demirköz ${ }^{2}$ L. Derome ${ }^{17}$ S. Di Falco ${ }^{39}$ V. Di Felice ${ }^{45}{ }^{\dagger}$ C. Díaz ${ }^{30}$ F. Dimiccoli ${ }^{40}$ P. von Doetinchem ${ }^{20}$ F. Dong ${ }^{35}$ F. Donnini ${ }^{45}{ }^{\dagger}$ M. Duranti ${ }^{37}$ A. Egorov ${ }^{10}$ A. Eline ${ }^{10}$ J. Feng ${ }^{10}$ E. Fiandrini ${ }^{37,38}$ P. Fisher ${ }^{10}$ V. Formato ${ }^{45, \dagger}$ C. Freeman ${ }^{20}$ Y. Galaktionov ${ }^{10}$ C. Gámez ${ }^{30}$ R. J. García-López ${ }^{27}$ C. Gargiulo ${ }^{15}$ H. Gast ${ }^{1}$ M. Gervasi ${ }^{32,33}$ F. Giovacchini ${ }^{30}$ D. M. Gómez-Coral ${ }^{20}$ J. Gong ${ }^{35}$ C. Goy ${ }^{3}$ V. Grabski ${ }^{31}$ D. Grandi ${ }^{32,33}$ M. Graziani ${ }^{37,38}$ S. Haino ${ }^{48}$ K. C. $\operatorname{Han}^{29}$ R. K. Hashmani ${ }^{2}$ Z. H. He ${ }^{19}$ B. Heber $^{26}$ T. H. Hsieh ${ }^{10}$ J. Y. Hu ${ }^{6,7}$ M. Incagli ${ }^{39}$ W. Y. Jang ${ }^{13}$ Yi Jia $^{10}$ H. Jinchi ${ }^{29}$ K. Kanishev ${ }^{40}$ B. Khiali ${ }^{45}{ }^{\dagger}$ G. N. Kim ${ }^{13}$ Th. Kirn ${ }^{1}$ M. Konyushikhin ${ }^{10}$ O. Kounina ${ }^{10}$ A. Kounine ${ }^{10}$ V. Koutsenko ${ }^{10}$ A. Kuhlman ${ }^{20}$ A. Kulemzin ${ }^{10}$ G. La Vacca ${ }^{32,33}$ E. Laudi ${ }^{15}$ G. Laurenti ${ }^{8}$ I.

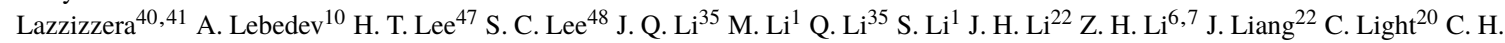
$\operatorname{Lin}^{48}$ T. Lippert ${ }^{24}$ J. H. Liu ${ }^{5}$ Z. Liu ${ }^{16}$ S. Q. Lu ${ }^{48}$ Y. S. Lu ${ }^{6}$ K. Luebelsmeyer ${ }^{1}$ J. Z. Luo ${ }^{35}$ Xi Luo ${ }^{23}$ S. S. Lyu ${ }^{19}$ F. Machate ${ }^{1}$ C. Maña $a^{30}$ J. Marín ${ }^{30}$ J. Marquardt ${ }^{26}$ T. Martin ${ }^{10,21}$ G. Martínez ${ }^{30}$ N. Masi ${ }^{8,9}$ D. Maurin ${ }^{17}$ A. Menchaca-Rocha ${ }^{31}$ Q. Meng ${ }^{35}$ V. V. Mikhailov ${ }^{34}$ D. C. Mo $^{19}$ M. Molero ${ }^{30}$ P. Mott ${ }^{10,21}$ L. Mussolin ${ }^{37,38}$ J. Negrete ${ }^{20}$ N. Nikonov ${ }^{1}$ F. Nozzoli ${ }^{40}$ A. Oliva ${ }^{8}$ M. Orcinha ${ }^{28}$ M. Palermo ${ }^{20}$ F. Palmonari ${ }^{8,9}$ M. Paniccia ${ }^{16}$ A. Pashnin ${ }^{10}$ M. Pauluzzi ${ }^{37,38}$ S. Pensotti ${ }^{32,33}$ H. D. Phan ${ }^{10}$ R. Piandani ${ }^{25}$ V. Plyaskin ${ }^{10}$ S. Poluianov ${ }^{36}$ X. Qin ${ }^{10}$ Z. Y. Qu ${ }^{48}$ L. Quadrani ${ }^{8,9}$ P. G. Rancoita ${ }^{32}$ D. Rapin ${ }^{16}$ A. Reina Conde ${ }^{27}$ E. Robyn ${ }^{16}$ S. Rosier-Lees ${ }^{3}$ A. Rozhkov ${ }^{10}$ D. Rozza ${ }^{32,33}$ R. Sagdeev ${ }^{11}$ S. Schael ${ }^{1}$ A. Schulz von Dratzig ${ }^{1}$ G. Schwering ${ }^{1}$ E. S. Seo ${ }^{12}$ Z. Shakfa ${ }^{2}$ B. S. Shan ${ }^{4}$ T. Siedenburg ${ }^{1}$ C. Solano ${ }^{10}$ J. W.

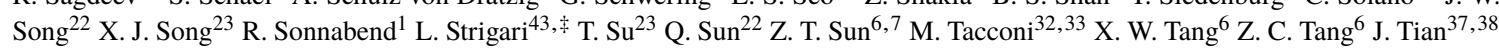
Samuel C. C. Ting ${ }^{10,15}$ S. M. Ting ${ }^{10}$ N. Tomassetti ${ }^{37,38}$ J. Torsti ${ }^{50}$ C. Tüysüz ${ }^{2}$ T. Urban ${ }^{10,21}$ I. Usoskin $^{36}$ V. Vagelli ${ }^{42,37}$ R. Vainio ${ }^{50}$ M. Valencia-Otero ${ }^{49}$ E. Valente ${ }^{43,44}$ E. Valtonen ${ }^{50}$ M. Vázquez Acosta ${ }^{27}$ M. Vecchi ${ }^{18}$ M. Velasco ${ }^{30}$ J. P. Vialle ${ }^{3}$ C. X. Wang ${ }^{22}$ L. Wang ${ }^{5}$ L. Q. Wang ${ }^{22}$ N. H. Wang ${ }^{22}$ Q. L. Wang ${ }^{5}$ S. Wang ${ }^{20}$ X. Wang ${ }^{10}$ Yu Wang $^{22}$ Z. M. Wang ${ }^{23}$ J. Wei $^{16}$ Z. L. Weng ${ }^{10}{\mathrm{H} . \mathrm{Wu}^{35} \text { R. Q. Xiong }}^{35}$ W. Xu ${ }^{22,23}$ Q. Yan ${ }^{10}$ Y. Yang ${ }^{46}$ I. I. Yashin ${ }^{34}$ H. Yi $^{35}$ Y. M. Yu ${ }^{19}$ Z. Q. Yu ${ }^{6}$ M. Zannoni ${ }^{32,33}$ C. Zhang ${ }^{6}$ F. Zhang ${ }^{6}$ F. Z. Zhang ${ }^{6,7}$ J. H. Zhang $^{35}$ Z. Zhang ${ }^{10}$ F. Zhao ${ }^{6,7}$ C. Zheng ${ }^{23}$ Z. M. Zheng ${ }^{4}$ H. L. Zhuang ${ }^{6}$ V. Zhukov ${ }^{1}$ A. Zichichi ${ }^{8}{ }^{8}$ N. Zimmermann ${ }^{1}$ and P. Zuccon ${ }^{40,41}$

${ }^{1}$ I. Physics Institute and JARA-FAME, RWTH Aachen University, 52056 Aachen, Germany. ${ }^{2}$ Department of Physics, Middle East Technical University (METU), 06800 Ankara, Turkey. ${ }^{3}$ Univ. Grenoble Alpes, Univ. Savoie Mont Blanc, CNRS, LAPP-IN2P3, 74000 Annecy, France. ${ }^{4}$ Beihang University (BUAA), Beijing, 100191, China. ${ }^{5}$ Institute of Electrical Engineering (IEE), Chinese Academy of Sciences, Beijing, 100190, China. ${ }^{6}$ Institute of High Energy Physics (IHEP), Chinese Academy of Sciences, Beijing, 100049, China. ${ }^{7}$ University of Chinese Academy of Sciences (UCAS), Beijing, 100049, China. ${ }^{8}$ INFN Sezione di Bologna, 40126 Bologna, Italy. ${ }^{9}$ Università di Bologna, 40126 Bologna, Italy. ${ }^{10}$ Massachusetts Institute of Technology (MIT), Cambridge, Massachusetts 02139, USA. ${ }^{11}$ EastWest Center for Space Science, University of Maryland, College Park, Maryland 20742, USA. ${ }^{12}$ IPST, University of Maryland, College Park, Maryland 20742, USA. ${ }^{13}$ CHEP, Kyungpook National University, 41566 Daegu, Korea. ${ }^{14}$ CNRIROE, 50125 Firenze, Italy. ${ }^{15}$ European Organization for Nuclear Research (CERN), 1211 Geneva 23, Switzerland. ${ }^{16}$ DPNC, Université de Genève, 1211 Genève 4, Switzerland. ${ }^{17}$ Univ. Grenoble Alpes, CNRS, Grenoble INP, LPSC-IN2P3, 38000 Grenoble, France. ${ }^{18}$ Kapteyn Astronomical Institute, University of Groningen, P.O. Box 800, 9700 AV Groningen, Netherlands. ${ }^{19}$ Sun YatSen University (SYSU), Guangzhou, 510275, China. ${ }^{20}$ Physics and Astronomy Department, University of Hawaii, Honolulu, Hawaii 96822, USA. ${ }^{21}$ National Aeronautics and Space Administration Johnson Space Center (JSC), Houston, Texas 77058, USA. ${ }^{22}$ Shandong University (SDU), Jinan, Shandong, 250100, China. ${ }^{23}$ Shandong Institute of Advanced Technology (SDIAT), Jinan, Shandong, 250100, China. ${ }^{24}$ Jülich Supercomputing Centre and JARA-FAME, Research Centre Jülich, 52425 Jülich, Germany. ${ }^{25}$ Institut für Experimentelle Teilchenphysik, Karlsruhe Institute of Technology (KIT), 76131 Karlsruhe, Germany. ${ }^{26}$ Institut für Experimentelle und Angewandte Physik, Christian-Alberts-Universität zu Kiel, 24118 Kiel, Germany. ${ }^{27}$ Instituto de Astrofísica de Canarias (IAC), 38205 La Laguna, and Departamento de Astrofísica, Universidad de La Laguna,38206 La Laguna, Tenerife, Spain. ${ }^{28}$ Laboratório de Instrumentação e Física Experimental de Partículas (LIP), 1649-003 Lisboa, Portugal. ${ }^{29}$ National ChungShan Institute of Science and Technology (NCSIST), Longtan, Tao Yuan, 32546, Taiwan. ${ }^{30}$ Centro de Investigaciones Energéticas, Medioambientales y Tecnológicas (CIEMAT), 28040 Madrid, Spain. ${ }^{31}$ Instituto de Física, Universidad Nacional Autónoma de México (UNAM), Ciudad de México, 01000 Mexico. ${ }^{32}$ INFN Sezione di MilanoBicocca, 20126 Milano, Italy. ${ }^{33}$ Università di MilanoBicocca, 20126 Milano, Italy. ${ }^{34}$ NRNU MEPhI (Moscow Engineering Physics Institute), Moscow, 115409 Russia. ${ }^{35}$ Southeast University (SEU), Nanjing, 210096, China. ${ }^{36}$ Sodankylä Geophysical Observatory and Space Physics and Astronomy Research Unit, University of Oulu, 90014 Oulu, Finland. ${ }^{37}$ INFN Sezione di Perugia, 06100 Perugia, Italy. ${ }^{38}$ Università di Perugia, 06100 Perugia, Italy. ${ }^{39}$ INFN Sezione di Pisa, 56100 Pisa, Italy. ${ }^{40}$ INFN TIFPA, 38123 Povo, Trento, Italy. ${ }^{41}$ Università di Trento, 38123 Povo, Trento, Italy. ${ }^{42}$ Agenzia Spaziale Italiana (ASI), 00133 Roma, Italy. ${ }^{43}$ INFN Sezione di Roma 1, 00185 Roma, Italy. ${ }^{44}$ Università di Roma La Sapienza, 00185 Roma, Italy. ${ }^{45}$ INFN Sezione di Roma Tor Vergata, 00133 Roma, Italy. ${ }^{46}$ National Cheng Kung University, Tainan, 70101, Taiwan. ${ }^{47}$ Academia Sinica Grid Center (ASGC), Nankang, Taipei, 11529, Taiwan. ${ }^{48}$ Institute of Physics, Academia Sinica, Nankang, Taipei, 11529, Taiwan. ${ }^{49}$ Physics Department and Center for High Energy and High Field Physics, National Central University (NCU),Tao Yuan, 32054, Taiwan. ${ }^{50}$ Space Research Laboratory, Department of Physics and Astronomy, University of Turku, 20014 Turku, Finland. *Also at Nikhef, 1098 XG Amsterdam, Netherlands. ${ }^{\dagger}$ Also at ASI Space Science Data Center (SSDC), 00133 Roma, Italy. ${ }^{\ddagger}$ Also at Policlinico S. Orsola-Malpighi, 40138 Bologna, Italy. 